\title{
Siesta poco reparadora
}

\author{
Javier Lucas Pérez-Romeroa, Francisco Hernández Fernández ${ }^{\mathrm{b}}$, Jorge García García \\ M. ${ }^{a}$ Belén González Ramírez ${ }^{\mathrm{c}}$ y Marta Lucas Pérez-Romero ${ }^{d}$
}

\begin{abstract}
${ }^{a}$ Médico de Familia.
Centro de Salud de La Roda. Gerencia de Atención Integrada de Albacete. España.

${ }^{\mathrm{b}}$ Especialista en Neurología. Gerencia de Atención Integrada de Albacete. España.

c Diplomada Universitaria en Enfermería. Centro de Salud de La Roda. Gerencia de Atención Integrada de Albacete. España. d Especialista en Psiquiatría. Gerencia de Atención Integrada de Almansa (Albacete). España.

Correspondencia:

Javier Lucas Pérez-Romero.

Centro de Salud de La Roda.

Correo electrónico:

jlucasp@sescam.jccm.es
\end{abstract}

Recibido el 22 de abril de 2014. Aceptado para su publicación el 31 de julio de 2014.

\section{RESUMEN}

Presentamos el caso de una mujer de 74 años de edad que estando previamente bien presenta de manera súbita dolor interescapular acompañado de alteración de la sensibilidad y la fuerza en miembro superior izquierdo.

Ante esta clínica se solicitó Resonancia Magnética Nuclear (RMN) que confirmó el diagnóstico de hematoma cervical epidural espontáneo.

Palabras Clave: Compresión medular. Hematoma cervical epidural espontáneo.

\section{ABSTRACT}

\section{A not very restful nap}

The present is the case of a 74 years old previously healthy woman who felt sudden interscapular pain along with strength and sensitivity impairment in her right arm. Facing these clinical symptoms, a Magnetic Resonance Imaging test (MRI) was performed which confirmed the diagnosis of spontaneous cervical epidural hematoma.

Key words: Spinal cord compression. Spontaneous cervical epidural hematoma.

\section{INTRODUCCIÓN}

Muchas son las situaciones clínicas que se presentan en la consulta de un Médico de Familia o en una consulta urgente hospitalaria que precisan una actuación rápida para solucionar el problema, pero pocas entidades clínicas precisan el uso urgente de una prueba complementaria que cuenta con escasas indicaciones en urgencias como es la RMN.

El caso que se plantea hace uso de esta prueba para confirmar la sospecha clínica inicial y plantear un diagnóstico y una actitud terapéutica apropiados.

\section{OBSERVACIONES CLÍNICAS}

Se trata de una mujer de 74 años, sin alergias farmacológicas, que como antecedentes médicos presentaba hipertensión arterial e hipotiroidismo bien controlado. No era diabética ni dislipémica conocida. Estaba diagnosticada de síndrome de apnea obstructiva del sueño (SAOS), en tratamiento con presión positiva continua en la vía aérea (CPAP). Fue ingresada en Cardiología en 2002 por angor inestable. Su tratamiento era AAS $100 \mathrm{mg}$ cada 24 horas, manidipino $10 \mathrm{mg}$ cada 24 horas, valsartán /hidroclorotiazida 80/12,5 cada 24 horas, nitroglicerina en parche, bromazepam 1,5 mg cada 12 horas y trimetazidina $20 \mathrm{mg}$ cada 24 horas.

Encontrándose previamente bien, la paciente se despierta de la siesta y, sin realizar ningún tipo de esfuerzo, sufre un cuadro de dolor súbito a nivel 
interescapular, punzante, irradiado a extremidad superior izquierda, acompañado de parestesias en miembro superior izquierdo y pérdida de fuerza en la mano izquierda. Acude a urgencias hospitalarias dos horas más tarde, momento en el que comienza a tener sacudidas involuntarias dolorosas de la pierna izquierda.

En la exploración física presentaba una tensión arterial de 174/82 mmHg, una frecuencia cardiaca de 55 latidos por minuto y una temperatura de 36,8 ${ }^{\circ} \mathrm{C}$. La paciente se encontraba con buen estado general, bien hidratada, bien perfundida, con buena coloración cutáneo-mucosa y eupneica. Los pulsos carotídeos eran rítmicos y simétricos, sin auscultarse soplos. No tenía adenopatías ni bocio. No presentaba ingurgitación yugular a $45^{\circ}$ ni rigidez de nuca. Los signos meníngeos fueron negativos. La auscultación cardiaca y la pulmonar no presentaron hallazgos. El abdomen era blando y depresible, sin masas ni megalias palpables y sin ser doloroso a la palpación. No presentaba edemas ni signos de trombosis venosa profunda. Los pulsos femorales y pedios estaban conservados y eran simétricos. En la exploración neurológica estaba consciente y orientada. La exploración de los pares craneales no presentaba focalidad. Lenguaje normal. Campimetría por confrontación normal. No extinción táctil.
Fuerza: paresia miembro superior izquierdo (proximal 4-/5, distal 2/5) y miembro inferior izquierdo (proximal 3/5, distal 2/5). Reflejos miotáticos: vivos generalizados con clonus aquíleo izquierdo inagotable y Hoffman en miembro superior izquierdo positivo. Babinski izquierdo. Espasmos involuntarios y dolorosos en flexión de miembro inferior izquierdo. Sensibilidad: hemihipoestesia izquierda. Coordinación normal.

El hemograma, la bioquímica y la coagulación eran normales. En el electrocardiograma se constató un ritmo sinusal a 55 latidos por minuto.

La clínica sugería compresión medular por lo que el diagnóstico diferencial que se planteó fue el que figura en la tabla 1. Ante la sospecha de compresión medular cervical se solicitó RMN cervical urgente (figura 1), la cual se informó como "colección extraaxial intradural posterior lateralizada hacia la izquierda a nivel de C3-C6, de aproximadamente 4 $\mathrm{cm}$, aunque su máximo grosor se localiza en C3-C5 de $7 \mathrm{~mm}$, es hiperintenso en T1 y T2 en relación a hematoma epidural agudo. A ese nivel también se visualiza una alteración de la señal de la médula espinal hiperintensa en T2 y STIR. Cervicoartrosis C3-C5 con disminución de los espacios intervertebrales".
Enfermedades raquídeas (vértebras, discos)
Espondiloartrosis cervical y lumbar.

Hernia discal.

Infecciones: osteítis, espondilitis.

Tumores vertebrales.

Traumas: fracturas y luxaciones.

Malformaciones congénitas: Síndrome de Klippel-Feil, malformaciones de la charnela occipito-cervical.

Tumores y quistes medulares intra y extradurales.

Infecciones epidurales, subdurales e intramedulares.

Hematomas epidurales.

Malformaciones vasculares.

Aracnoiditis.

Infarto medular.

Mielitis transversa. 


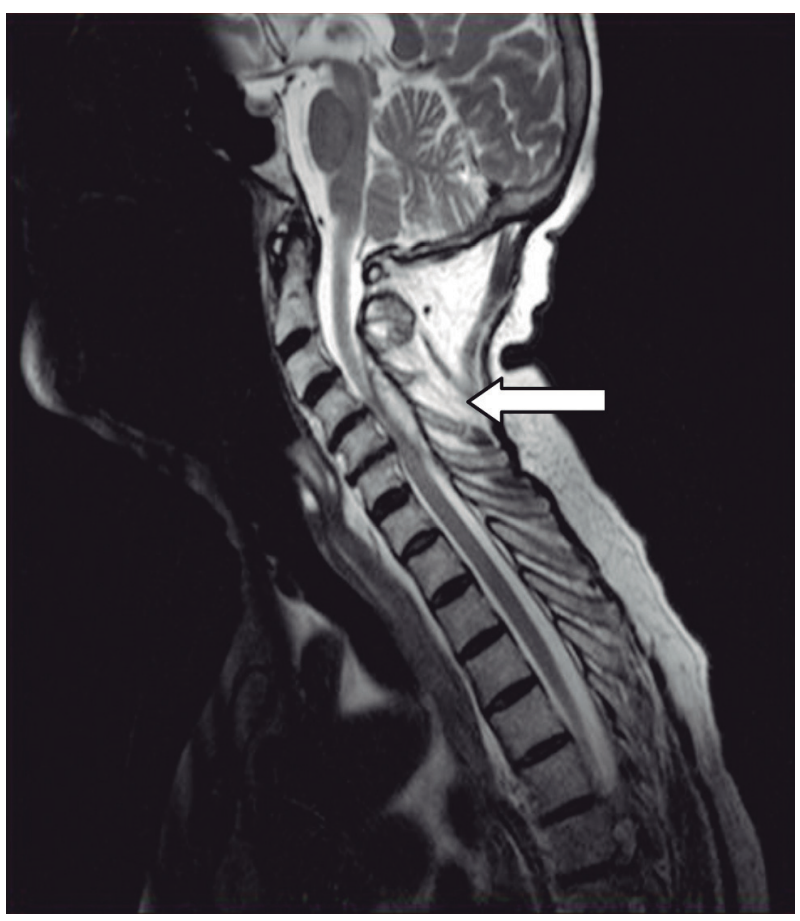

Figura 1. RMN del día que acudió a Urgencias

Tras confirmarse el diagnóstico de hematoma espinal se solicitó valoración por neurocirugía, que recomendó tratamiento conservador debido a la rápida recuperación neurológica.

Durante su ingreso en planta mejoró progresivamente manteniendo al alta hemiparesia izquierda 5-/5 y una discreta torpeza en la marcha, aunque autónoma. Desapareció el trastorno sensitivo y los movimientos involuntarios. Como única incidencia presentó un episodio de presíncope con frecuencia cardiaca de 35 latidos por minuto y tensión arterial de 70/35 por lo que se solicitó consulta a cardiología que realizó un Holter en el que resultó un trazado en ritmo sinusal con frecuencias medias de 45 Ipm, máximas de 50 y mínimas de 35 sin ninguna pausa patológica detectada por lo que diagnostican el episodio como presíncope vasovagal y recomiendan seguimiento ambulatorio.

Por parte de neurología al apreciar evolución favorable en RMN tras 8 días de hospitalización (figura 2), se le da el alta hospitalaria y se programa nueva RMN ambulatoria en dos meses para control.

\section{COMENTARIOS}

El hematoma epidural espinal espontáneo es una patología muy infrecuente, estimándose una incidencia de 1 caso por cada millón de habitantes.

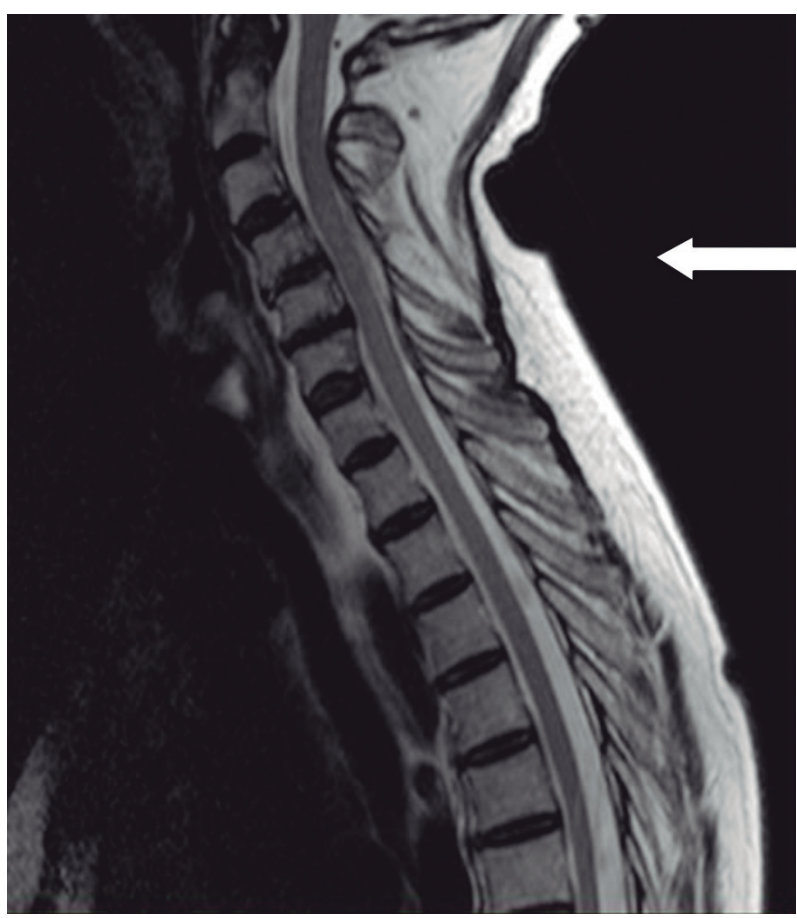

Figura 2. RMN de control a los 8 días de su atención en urgencias y previa al alta hospitalaria

Representa el 0,3-0,6\% de todas las lesiones espinales epidurales ocupantes de espacio. Las etiologías más frecuentes son: neoplasias, coagulopatías, terapias anticoagulantes, vasculitis sistémicas como la poliarteritis nodosa, malformaciones vasculares y enfermedad de Paget ${ }^{1}$.

La clínica habitual consiste en dolor de inicio agudo, asociado a veces a parestesias radiculares, evolucionando a veces en minutos u horas a signos de compresión medular progresiva².

El diagnóstico se debe hacer con RMN urgente, que nos permita apreciar el grado de compromiso de la médula y la etiología del mismo, siendo esta sospecha una de las escasas indicaciones de RMN urgente ${ }^{3}$. Esta indicación se deriva de la inmediata intervención neuroquirúrgica que precisa en determinados casos ya que la lesión puede ser reversible con tratamiento apropiado y un retraso en el mismo podría ocasionar un déficit neurológico irreversible. La laminectomía para evacuar el hematoma y la descompresión medular precoz es el tratamiento de elección, pero a veces la clínica es pobre y no evoluciona o incluso recupera tempranamente, por lo que se opta por un tratamiento conservador acompañado de exámenes neurológicos seriados con RMN de control ${ }^{4}$. 


\section{BIBLIOGRAFÍA}

1. Iriarte García-Baquero LM, Fernández-Bolaños Porras R, Galán Barranco J, Friera Acebal G. Enfermedades que causan compresión medular. Medicine.1998;7(97):4503-4511.

2. Márquez Rivas J. Procedimientos terapéuticos en el hematoma subdural y epidural. Medicine. 2007;9(73):4708-4711.
3. Aparici F, Más F, Solera MC, Moro G. Hematoma epidural cervical espontáneo: a propósito de un caso y revisión de la bibliografía. Radiología. 2002;44(3):118-121.

4. Alday Muñoz E, Maseda E, Gutierrez L, Galván B. Hematoma epidural secundario a catéter epidural para analgesia en un paciente politraumatizado. Med Intensiva. 2006;30(1):30-2. 